\title{
¿CÓMO PERCIBEN LAS UNIVERSIDADES EL FOMENTO DEL EMPRENDIMIENTO?
}

Mg. Emma Pérez Palacios*

eeperezpalacios@yahoo.com

\begin{abstract}
RESUMEN
El presente artículo muestra una parte de los resultados del estudio "Fomento del Emprendimiento en los Jóvenes Universitarios 2010 Este estudio recoge los aportes de nueve centros de formación superior y cinco instituciones públicas y privadas vinculadas al fomento del emprendimiento. En esta oportunidad presentamos la posición de los centros de formación superior respecto a la importancia de fomentar el emprendimiento. El estudio revela que el fomento del emprendimiento es una acción considerada como importante; a pesar de esta clasificación, los centros de formación superior no están usando este tema como estrategia de diferenciación y posicionamiento en el mercado. Asimismo, se identifica que las principales razones por las que los jóvenes estudiantes de los centros de formación superior no se ven atraídos por el desafío emprendedor son: a) las expectativas sobre crecimiento económico del país, producto del cual el mercado laboral dependiente requeriría mano de obra calificada en determinados sectores, b) temor al riesgo, c) desconocimiento sobre cómo y en qué sector iniciar una empresa, y d) falta de capital
\end{abstract}

Palabras Claves: Emprendimiento, cultura emprendedora, la universidad y la creación de empresas

\begin{abstract}
This paper presents some of the results of the "Promotion of Youth Entrepreneurship in College" 2010. This study reports the contributions of 9 centers of higher education and 5 public and private institutions linked to the promotion of entrepreneurship. In this opportunity we present the position of the centers of higher education regarding the importance of promoting entrepreneurship. The study reveals that the promotion of entrepreneurship is an action considered IMPORTANT, despite this classification, the top training centers are not using this issue as a strategy of differentiation and market positioning. It also identifies the main reasons why young students of higher education centers are not attracted to the entrepreneurial challenge are: a) expectations about economic growth in the country, a product which would require the labor market labor dependent skilled labor in certain sectors, b) risk aversion, c) lack of knowledge about how and in which sector to start a business, and d) lack of capital.
\end{abstract}

Keywords: entrepreneurship, entrepreneurshi culture, university and bussines maceration

\footnotetext{
* Docente de la Facultad de ciencias Administrativas de la UNMSM, Magister en Administración, Licenciada en estadística y Especialista en Investigación.
} 


\section{INTRODUCCIÓN}

Según el Censo Universitario 2010, de cada 100 jóvenes en pre grado 46 declaran que existen cursos de emprendimiento en su universidad, 30 consideran que no existen dentro de su formación y 23 no saben.

Esto evidencia que el fomento del emprendimiento entre los jóvenes estudiantes de los centros de formación superior (universidades) es un camino que se está empezando a recorrer en el país.

Las iniciativas para fomentar el emprendimiento desde los centros de formación superior son muchas y muy variadas, dependiendo del objetivo que cada universidad persiga, la gran mayoría aporta al componente motivacional, otros al desarrollo de las aptitudes emprendedoras, es decir a la elaboración de buenos documentos que sustenten la idea de negocio (planes de empresa o proyectos empresariales) y otro grupo se enfoca en la actitud del joven que está detrás de la idea.

La UNMSM no es ajena a este contexto, por lo que ha asumido como un reto el desarrollo emprendedor de sus estudiantes de un modo integral, en el que no sólo se tome en cuenta los rasgos de personalidad sino los valores que marcarán su manera de actuar, lo que significa aprovechar las lecciones derivadas de las investigaciones y la docencia y traducirlas en planteamientos específicos orientados al desarrollo sostenible del país.

\section{MÉTODOS}

La investigación realizada recoge información cualitativa. Se utilizó la técnica de la entrevista, a través de cuestionarios estructurados dirigidos a dos grupos de discusión claramente identificados y diferenciados:

Grupo 1: Representantes de 9 Centros de Formación Superior (universida des e institutos).

Grupo 2: Representantes de 5 Instituciones públicas y privadas comprometidas con el fomento del emprendimiento.

Así tambien se clasifica como una investigación del tipo descriptiva, porque describe en qué medida los centros de formación superior están fomentando la cultura emprendedora de sus estudiantes y cómo a decir de los expertos y entendidos en el fomento del emprendimiento perciben la labor de las universidades en ésta noble labor.

Así mismo, se describe las políticas y actividades de desarrollo emprendedor que realizan los centros de formación superior públicos y privados, así como otras instituciones comprometidas en el tema de emprendimiento, como ONG e instituciones públicas, para lo cual se hace uso de fuentes primarias y secundarias de información. Es preciso volver a destacar que en el presente artículo se muestran los resultados obtenidos sólo del grupo 1.

\section{RESULTADOS}

1. La Promoción del emprendimiento en el Perú.

A pesar del gran número de universidades existentes a nivel nacional, son muy pocas las que promueven el espíritu emprendedor en sus estudiantes, aquellas universidades que sí lo hacen, para cumplir con dicho fin, han creado instancias universitarias dedicadas al emprendimiento e incubación de empresas, a continuación, se describen algunas de ellas:

- Universidad ESAN: Cuenta con el Centro de Desarrollo Emprendedor, a través del cual están promoviendo el concurso nacional universitario del talento emprendedor, cuyo objetivo es promover el espíritu emprendedor de los jóvenes universitarios de todas las facultades y especialidades de todas las universidades del Perú, que se encuentren cursando entre el sétimo y décimo ciclo de estudios.

- Pontificia Universidad Católica del Perú: a través del Centro de Innovación y Desarrollo Emprendedor-CIDE, se dedica a la promoción de la cultura emprendedora dentro y fuera de la comunidad universitaria. El CIDE promueve la creación y el desarrollo de empresas que aprovechen la formación y conocimientos desarrollados en la PUCP para la generación de bienes y servicios con valor agregado, y apoya la introducción de los mismos en los mercados nacional e internacional.

- Universidad de Piura: cuenta con la Incubadora de base tecnológica e innovación - IncubaUdeP: tiene como objetivo promover el espíritu emprendedor y la innovación de los alumnos, egresados y tesistas de la universidad, apoyando a los que tengan ideas de negocio en el campo tecnológico. Brinda asesoría y acompa- 
ñamiento constantes para el emprendedor, de manera que su idea pueda convertirse primero en un plan de negocios coherente y, luego, en una pequeña empresa autosostenible.

- Universidad San Martín de Porres: trabaja a través del Instituto de Innovación y Promoción para el Emprendimiento Universitario - IPPEU; surge en la actual coyuntura nacional, para promover una cultura emprendedora y empresarial, apoyando a sus alumnos en la formación de empresas sostenibles, para generar desarrollo y empleo sostenible.

- Universidad Continental: Ha creado la Incubadora de Empresas CONTI INCUBA, como un Programa Especial encargada de fortalecer las capacidades y habilidades emprendedoras de sus estudiantes y egresados, brindándoles herramientas necesarias, para generar ideas y empresas potenciales e innovadoras que le permitan lograr un nivel competitivo en el mercado.

Por otro lado, existen instituciones privadas que vienen promoviendo el emprendimiento como:

- La Asociación Pro Bienestar y Desarrollo-PROBIDE, quienes año a año ponen en marcha el programa nacional de jóvenes emprendedores "creer para crear", que bajo la modalidad de un concurso de planes de negocio, apoyan a la creación de nuevas empresas.

- El Centro de Transferencia Tecnológica a Universitarios - CTTU San José es una Asociación Civil sin fines de lucro, promovida por La Compañía de Jesús. Inicia sus actividades desde el año 1991, con el objeto de desarrollar iniciativas empresariales en sectores de bajos ingresos para contribuir a mejorar sus condiciones de vida en los valles de la costa de La Libertad.

- La Incubadora de Empresas de Base Tecnológica del INICTEL-UNI se desenvuelve en el campo de las Tecnologías de la Información y Comunicación (TIC), por lo que está orientada a que los emprendedores desarrollen ideas innovadoras ofreciéndoles infraestructura y servicios diferenciados que permitan crear un ambiente de trabajo adecuado para el desarrollo de sinergias entre sus empresas.

Asimismo, las organizaciones privadas promueven el emprendimiento a través de concursos para emprendedores:

- La empresa NESTLE promueve el concurso "Despertando Ideas" con el objeto de reconocer, valorar y desarrollar el potencial de los jóvenes universitarios, a través de la elaboración de proyectos empresariales de diferente índole tales como micro, pequeñas y medianas empresas así como proyectos de desarrollo social.

- El Desafío SEBRAE PERU es un juego de simulación de gestión de empresas, dirigido a estudiantes de cualquier especialidad, que a la fecha de la inscripción, se encuentren matriculados de manera regular en instituciones de enseñanza superior, (universidades o institutos superiores) debidamente acreditadas por el Ministerio de Educación, sin existir ninguna limitación respecto a su edad, curso o período. El juego utiliza un software exclusivo y tiene como objetivo perfeccionar los conocimientos del área de negocios de todos los participantes, independientemente de la carrera que estén cursando

2. Los centros de formación superior-CFS, consideran importante fomentar el emprendimiento empresarial y la creación de empresas entre sus estudiantes; el sustento para ello se basa en dos razones válidas:

- El emprendimiento es una opción de realización del joven, es una manera válida de lograr que los jóvenes construyan su propia empleabilidad, y logren su crecimiento personal y profesional.

- La empresa es el motor del crecimiento del país, los CFS consideran que la empresa es el agente de cambio en las sociedades modernas, por lo tanto, la existencia de más empresas con sentido de responsabilidad redundará en el desarrollo local, regional y nacional. Por lo que también es importante fortalecer el vínculo empresa-academia.

3. Los CFS vienen desarrollando -en distinto grado- actividades de fomento del emprendimiento, en función a sus recursos y su conocimiento del tema. Dichas actividades pueden clasificarse en dos grupos: Extracurriculares y Curriculares.

4. Las Actividades Extracurriculares están referidas a aquellas que se desarrollan fuera del plan de estudios "formal"; pueden ser 
a. Actividades generales realizadas por alguna facultad o escuela profesional, dirigidas a motivar y despertar el interés de los estudiantes (charlas y/o seminarios sobre historias de éxito, casos de empresarios más destacados), no se profundiza demasiado en el aspecto formativo del joven interesado en la creación de empresa.

b. Actividades específicas realizadas desde una instancia creada dentro de los CFS de manera exclusiva para el fomento del emprendimiento en los estudiantes.

c. El nivel de profundidad en el fomento del emprendimiento depende de las acciones que realizan, las mismas que se pueden clasificar en 3 niveles: Nivel 1, Motivacional; charlas y/o seminarios sobre historias de éxito, casos de empresarios más destacados). Nivel 2, Preparación; cursos y talleres para identificar ideas de negocio y elaborar planes de negocio, diseño y participación en concursos empresariales. Nivel 3, Puesta en marcha; acciones de seguimiento a la gestión de los negocios que se estén implementando.

d. Los CFS no muestran mucha evidencia respecto a la labor realizada en el nivel 3, comparado con el trabajo realizado en el nivel 1 , mientras tanto algunas instituciones se han preocupado por intervenir en el nivel 2.

5. Las actividades curriculares, son aquellas que se desarrollan en el marco del plan de estudios de cada carrera profesional, como parte de la formación académica de los jóvenes estudiantes.

Los representantes de los centros de formación superior entrevistados afirman realizar actividades curriculares que fomentan el emprendimiento, a través de cursos implementados para tal fin, como generación de empresas, creatividad empresarial, introducción a los negocios, plan de negocios, liderazgo y trabajo en equipo, presentaciones efectivas, emprendimiento, iniciativa empresarial, realidad empresarial peruana.

Actividades Curriculares que fomentan el emprendimiento

\begin{tabular}{|c|c|c|}
\hline Ciclo & Cursos que fomentan el emprendimiento & Objetivo del curso \\
\hline $1^{\mathrm{o}}$ & Introducción a los negocios & Manejar conceptos básicos de empresa \\
\hline 10 & Presentaciones efectivas & $\begin{array}{c}\text { Desarrollar la actitud emprendedora en } \\
\text { el alumno }\end{array}$ \\
\hline $2^{\mathrm{o}}$ & Liderazgo y trabajo en equipo & $\begin{array}{c}\text { Desarrollar las capacidades de trabajo } \\
\text { en equipo }\end{array}$ \\
\hline $6^{0}$ & Creatividad empresarial & Desarrollar habilidades Innovadoras \\
\hline 60 & Iniciativa empresarial & $\begin{array}{l}\text { Generar e implementar planes de nego- } \\
\text { cios }\end{array}$ \\
\hline $7^{0}$ & Realidad empresarial peruana & $\begin{array}{c}\text { Incentivar al alumno a crear su propia } \\
\text { empresa }\end{array}$ \\
\hline $7^{0}, 8^{\circ}, 9^{\circ} \underline{o}$ & plan de negocios I, II y III. & Elaborar un plan de negocio viable \\
\hline 90 & Generación de empresas & Aprender a elaborar planes de negocios \\
\hline $10^{\mathrm{o}}$ & Emprendimiento & $\begin{array}{c}\text { Conocer las competencias propias del } \\
\text { empresario y desarrollar la idea del } \\
\text { negocio a través de la elaboración de un } \\
\text { Plan de Negocio }\end{array}$ \\
\hline
\end{tabular}


a. De los centros de formación superior entrevistados, el que le dedica más atención al fomento del emprendimiento manifiesta que el $17 \%$ del total de cursos están orientados a este fin, lo que totaliza 527 horas en la formación del futuro profesional.

b. Los cursos implementados por los centros de formación superior tienen las siguientes características:

i. Los primeros ciclos están orientados al nivel 1, es decir, tratan de motivar y mostrar al joven estudiante la opción de la creación de empresas como un camino de desarrollo personal y profesional.

ii. Los últimos ciclos se orientan al nivel 2, donde los futuros profesionales desarrollan sus capacidades técnicas de cómo elaborar un buen plan de empresa, reciben todos los conocimientos y se preparan para cuando se decidan a crear empresas.

a. Otra característica de los centros de formación superior es que, aquellos que trabajan a nivel motivacional, no lo hacen a nivel de preparación técnica y viceversa. Es decir, aún no hay una correlación clara en el fomento del emprendimiento de manera integral, que cubra al menos los niveles 1 y 2 .

c. El nivel 3, referido a la puesta en marcha de los negocios no es trabajado con actividades curriculares, ya que esta etapa depende íntegramente de la voluntad del joven estudiante, siempre que realmente quiera iniciar una actividad empresarial como opción de realización personal y profesional.

d. Esto no significa que los centros de formación superior no puedan hacer actividades del nivel 3 dentro de la currícula educativa, por el contrario, sí es posible apoyar la puesta en marcha de los nuevos negocios de los jóvenes, siempre que se considere dentro de cursos o créditos "electivos", elegidos libremente por los estudiantes.

e. La entrevista no arroja información respecto a la posibilidad de graduarse y obtener el título técnico o profesional con un plan de negocio o plan de empresa puesto en marcha y/o mejor aún, con una empresa en funcionamiento, producto de su paso por los cursos de fomento del emprendimiento.
6. Las principales razones por las que el joven universitario no se ve atraído por el desafío emprendedor son:

a. Los representantes de los centros de formación superior opinan que los jóvenes estudiantes están preparados para elaborar planes y proyectos empresariales y que además estarían interesados en hacerlos realidad, sin embargo, no lo hacen porque consideran que deben mejorar otros factores externos para que los jóvenes se "animen" a hacer empresa.

b. Desde la experiencia de los representantes de los centros de formación superior, en promedio, de cada 10 alumnos, 7 optan por un empleo dependiente y 3 se inclinan por crear su propia empresa. Las razones por las que los jóvenes optan por un empleo dependiente antes que crear su propia empresa y ser su propio jefe son:

- Las expectativas de crecimiento económico, hace que la creación de empresas no sea la primera opción entre los jóvenes, principalmente de las carreras vinculadas al crecimiento de la economía (como comercio exterior, negocios internacionales, turismo, gastronomía, etc.), debido a que se percibe un aumento por la demanda por profesionales calificados que se estarían insertando al mercado laboral dependiente.

- Temor al riesgo; la inseguridad y el miedo a los cambios, que hacen que la estabilidad laboral sea una opción muy apreciada y valorada, inclinan la balanza hacia el empleo dependiente.

- Desconocimiento sobre cómo y en qué sector iniciar una empresa; falta de un mayor apoyo y orientación a los interesados en crear empresa.

- Falta de capital; por lo que muchos prefieren conseguir un empleo dependiente hasta juntar el monto necesario para iniciar su propia empresa.

7. A pesar de esta realidad y los motivos por los que el empleo dependiente es más valorado, los entrevistados consideran que sí es posible incrementar la preferencia de los estudiantes por la creación de empresas como opción de realización personal y profesional. Asimismo, consideran que sus centros educativos lo pueden hacer a través de diversos medios como son: 
a. Implementando o fortaleciendo las incubadoras de empresas.

b. Sensibilizando a los estudiantes sobre la realidad del mercado laboral

c. A través de la ejecución y participación más activa en actividades como concursos para emprendedores, además de la formación de consultores especializados en la atención a emprendedores.

d. Fortaleciendo las redes sociales y redes de apoyo con instituciones públicas y privadas con experiencia en el trabajo con emprendedores.

8. Las instituciones educativas opinan que hay factores del entorno que se deben mejorar a favor de los emprendedores:

a. Apoyo para la implementación de incubadoras de empresas.

b. Fuentes de financiamiento para los proyectos de los emprendedores a nivel de capital semilla o capitales ángeles.

c. Mejorar el acceso a la información respecto a mercados y créditos.

d. Facilitar y flexibilizar la formalización de las empresas, y el sistema tributario para nuevas empresas.

e. Mayor vinculación del Estado con la academia y el sector empresarial.

9. Por otro lado, las principales limitantes que tienen los emprendedores para iniciar una empresa a decir de los representantes de los centros de formación superior son (en orden de importancia):

a. Desconocimiento de la gestión de negocios,

b. Desconocimiento del mercado a atender

c. Falta de capital

d. Poca experiencia en el rubro.

\section{CONCLUSIONES}

1. Las universidades reconocen la importancia de fomentar el emprendimiento desde el sistema educativo superior, pero llama la atención que las acciones que se vienen realizando no logran consolidar una intervención efectiva que se traduzca en la creación de nuevas empresas desde las universidades.
2. Las universidades deben realizar actividades curriculares y extracurriculares a fin de complementar la intervención; en la medida que el emprendimiento en una cuestión de voluntad y de libre elección, principalmente en la etapa de puesta en marcha de los nuevos negocios, se puede plantear la posibilidad de desarrollar esta etapa dentro de la currícula educativa siempre que se maneje como un curso electivo.

3. Para fortalecer las actividades curriculares, las universidades - y en especial la UNMSM - debe desarrollar los conocimientos y las capacidades de sus catedráticos para manejar una estrategia única de intervención que vincule las acciones de motivación, preparación y puesta en marcha de nuevos negocios.

4. Si bien es cierto, la mayoría de estudiantes universitarios tienen los conocimientos técnicos para desarrollar un proyecto empresarial, éstos mismos jóvenes no tienen el nivel de motivación necesario como para asegurar la inclinación por el emprendimiento como opción de realización personal y profesional.

5. El trabajo motivacional debe ser constante, es decir, si bien es cierto, la promoción del emprendimiento se inicia con acciones de motivación, estas acciones se deben realizar a lo largo del proceso de preparación y durante los primeros pasos de puesta en marcha de los nuevos negocios.

6. Es fundamental fortalecer las redes de apoyo entre la universidad y las instituciones públicas y privadas que promueven el emprendimiento, de este modo se podría suplir las carencias o desconocimientos respecto al tema promocional.

\section{LITERATURA CITADA}

Asamblea Nacional de Rectores; Resultados del II Censo Universitario 2010.

Asociación Peruana de Incubadoras de Empresas, Perú Incuba www.peruincuba.net

Dirección Nacional de la Micro y Pequeña Empresa - Ministerio de Trabajo y Promoción del Empleo, Elaboración de Estadísticas de la Micro y Pequeña Empresa, 2006

Dirección Nacional de la Micro y Pequeña Empresa - Ministerio de Trabajo y Promoción del Em- 
pleo, Directiva № 001-2008-MTPE/3.11.3, Ejecución de acciones para el fomento del emprendimiento con enfoque de oportunidad a nivel regional, 2008

Linares, Dino - Colectivo Integral de Desarrollo, en estudio elaborado para el Ministerio de Trabajo y Promoción del Empleo: "Creación de Empresas y apoyo a Emprendedores en el Perú", Lima 2002.
Sub Secretaría de PYME - Secretaría de Economía del Estado de México, Sistema Nacional de Incubadoras de Empresas, SNIE www.contactopyme.gob.mx/snie/PrinciplSNIE.asp

Tefarikis, Elías: Revista América Economía Artículo "Educación para el emprendimiento - Chile", México 2009

Wompner, Fredy: "Un modelo de incubadora de negocios universitaria; en la ruta de la innovación organizacional", Chile 2007. 